\title{
Preoperative sleep quality affects postoperative pain and function after total joint arthroplasty: a prospective cohort study
}

\author{
Ze-Yu Luo ${ }^{\dagger}$, Ling-Li Li ${ }^{\dagger}$, Duan Wang, Hao-Yang Wang, Fu-Xing Pei and Zong-Ke Zhou* (D
}

\begin{abstract}
Background: The relationship between preoperative sleep quality and postoperative clinical outcomes after total joint arthroplasty (TJA) is unclear. We performed a prospective cohort study to determine whether preoperative sleep quality was correlated with postoperative outcomes after TJA.

Methods: In this prospective cohort study, 994 patients underwent TJA. Preoperative sleep measures included scores on the Pittsburgh Sleep Quality Index (PSQI), the Epworth Sleepiness Scale (ESS), and a ten-point sleep quality scale. The primary study outcome measured was the visual analog scale (VAS) pain score to 12 weeks postoperation. The consumption of analgesic rescue drugs (oxycodone and parecoxib) and postoperative length of stay (LOS) were recorded. We also measured functional parameters, including range of motion (ROM), Knee Society Score (KSS), and Harris hip score (HHS).

Results: The mean age for total knee and hip arthroplasties was 64.28 and 54.85 years, respectively. The PSQI scores were significantly correlated with nocturnal and active pain scores and ROM and functional scores from postoperative day 1 (POD1) to POD3. In addition, significant correlation was noted between the correlation between the active pain scores and ESS scores in the TKA group at postoperative 3 months. The consumption of analgesics after joint arthroplasty was significantly correlated with the PSQI scores. Moreover, significant correlations were noted between the sleep parameters and postoperative length of hospital stay (LOS).
\end{abstract}

Conclusion: Preoperative sleep parameters were correlated with clinical outcomes (i.e., pain, ROM, function, and LOS) after TJA. Clinicians should assess the sleep quality and improve it before TJA.

Keywords: Total joint arthroplasty, Pain, Sleep, Clinical function

\section{Introduction}

More than 21 million adults in the US have osteoarthritis (OA) [1]. These patients experience a worsening in their quality of life and a decreased ability to perform activities of daily living [2]. Studies have reported that patients with OA have decreased sleep quality in association with increased night pain, which correlates with radiographic severity of disease [3, 4]. Pain has been

\footnotetext{
* Correspondence: zhouzongke@scu.edu.cn

†Ze-Yu Luo and Ling-Li Li contributed equally to this work.

Department of Orthopedics, West China Hospital/West China School of Medicine, Sichuan University, 37\# Wuhou Guoxue Road, Chengdu 610041 People's Republic of China
}

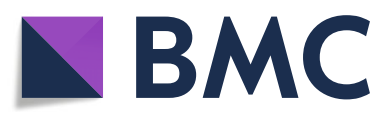

(c) The Author(s). 2019 Open Access This article is distributed under the terms of the Creative Commons Attribution 4.0 International License (http.//creativecommons.org/licenses/by/4.0/), which permits unrestricted use, distribution, and reproduction in any medium, provided you give appropriate credit to the original author(s) and the source, provide a link to the Creative Commons license, and indicate if changes were made. The Creative Commons Public Domain Dedication waiver (http://creativecommons.org/publicdomain/zero/1.0/) applies to the data made available in this article, unless otherwise stated.

identified as a cause of poor sleep quality. Poor sleep quality has been associated with increased pain perception [5].

Total joint arthroplasty (TJA) has proven to be a surgical option for patients with advanced osteoarthritis in terms of improved function and pain relief [6]. Both primary total hip (THA) and knee (TKA) arthroplasties can relieve pain, restore function, and increase mobility to levels observed in the general population [7]. However, a previous study has shown that patients' sleep quality is impaired for 3 months after joint replacement and further affects patients' rapid recovery after surgery [8]. 
Several studies have investigated the influence of different interventions on postoperative sleep quality [9-11]. The relationship between postoperative sleep quality and postoperative rehabilitation and did not further explore the influence of preoperative sleep quality in patients. Although many previous studies have investigated the influence of postoperative sleep quantity on rehabilitation after total joint replacement, the correlations between the preoperative sleep quality and postoperative clinical outcomes (i.e., pain and function) are unclear. Studies of healthy, pain-free volunteers have consistently shown that sleep continuity disturbance impairs endogenous pain-inhibitory function and increases spontaneous pain, supporting a possible pathophysiologic role of sleep disturbance in pain [12-14]. One study found that a presurgery extended time-in-bed and the associated increase in sleep time in short-sleeping patients scheduled for undergoing joint replacement resulted in reduced postsurgery pain ratings and opioid medication use [15]. However, they included only seven patients in the treatment group, which may not be powered to detect a significant difference.

We, therefore, performed a prospective cohort study to determine whether presurgery sleep quality was correlated with postoperative pain, function, complications, and length of hospital stay (LOS) after TKA and THA.

\section{Methods}

Approval was obtained from the Clinical Trials and Biomedical Ethics Committee of West China Hospital, and written informed consent was obtained from all participants. This study was registered in the Chinese Clinical Trial Registry (ChiCTR1900022357).

\section{Patients}

From March 2017 to March 2018, patients who were scheduled to undergo primary unilateral TJA were eligible for study enrollment. Patients were excluded based on the following criteria: primary sleep disorders (insomnia, apnea, and restless legs), mental disorders (depression, anxiety), chronic uncontrolled medical illnesses beyond their joint disease (preoperative hepatic or renal dysfunction and serious cardiac and/or cerebrovascular comorbidities), planned revision surgery, bilateral arthroplasty, and refusal to participate.

\section{Surgical procedure and pain management}

All total hip and knee replacement surgeries were performed under general anesthesia by two orthopedic surgeons. The patients were sent to the inpatient orthopedic unit where they spent 3-4 days before being discharged to continue their recovery at home after the surgery. The lights in the inpatient unit were dimmed between $10 \mathrm{pm}$ and $6 \mathrm{am}$, and patient doors were closed.
Six beds were placed in each inpatient unit. No attempt was made to manage the sleep quality for this group of patients.

Pain management [16] was used for pain control in all patients according to the standard practice at our institution, as described by Lei et al. [17]. Multimodal oral analgesic drugs (diclofenac $50 \mathrm{mg}$ q $12 \mathrm{~h}$, Novartis Biomedical Research Co., Ltd., Switzerland, and pregabalin 75 mg q $8 \mathrm{~h}$, Pfizer Pharmaceuticals Co., Ltd., USA) were administered for preemptive analgesia 1 day before surgery. This analgesic regimen was repeated when patients resumed postoperative oral intake. When patients reported pain greater than 4 on a $0-10$ visual analog scale (VAS), oral oxycodone (10 mg q $8 \mathrm{~h}$ ) was utilized. An intramuscular injection of parecoxib $(40 \mathrm{mg})$ was used if a patient claimed severe pain greater than 6 on the VAS [17].

Prophylaxis against venous thromboembolism was administered following the standard practice at our institution $[18,19]$. An examination of the clinical symptoms of deep vein thrombosis (DVT) in all patients was conducted according to the standard practice at our institution [18]. During hospitalization, the patients were examined daily for any clinical symptoms of DVT. Ultrasound examinations were also performed routinely at specific time points (preoperatively, at discharge, and at the 2-week and 3month follow-ups). If a patient was symptomatic, an ultrasound examination was performed immediately. A contrast-enhanced chest spiral computed tomography was performed immediately for any clinical signs of pulmonary embolism (PE) $[18,20]$. In addition, other complications including superficial infection, hematoma, and wound secretion were also diagnosed according to the standardized list and definitions [21, 22].

\section{Sleep quality assessments}

Sleep quality was evaluated by 2 separate assessments (ZY. L. and D. W.). Sleep quality can be measured by using three subjective methods. The patients were required to complete the Pittsburgh Sleep Quality Index (PSQI), a self-rated questionnaire that assesses sleep quality and disturbances over a 1-month time interval prior to admission [9, 23]. Nineteen individual items generate 7 "component" scores: subjective sleep quality, sleep latency, sleep duration, habitual sleep efficiency, sleep disturbances, use of sleeping medication, and daytime dysfunction. The sum of scores for these 7 components yields 1 global score, with a maximum score of 21 . A higher score indicates worse sleep dysfunction, and a score greater than 5 indicates poor sleep quality [8]. Daytime sleepiness assessment was assessed using the Epworth Sleepiness Scale (ESS) [24]. The ESS is a standardized, validated questionnaire for assessing daytime sleepiness. It contains 8 questions that ask respondents to assess on a 4-point scale the typical opportunities for 
them to fall asleep or doze off during various daily activities. The Epworth sleep scale score ranged from 0 to 24 (sum of 8 scores, $0-3$ ), with higher scores indicating a greater propensity for daytime sleepiness. In general, ESS scores can be interpreted as follows: $0-5$ lower normal daytime sleepiness, 6-10 higher normal daytime sleepiness, 11-12 mild excessive daytime sleepiness, 1315 moderate excessive daytime sleepiness, and 16-24 severe excessive daytime sleepiness [25]. Finally, we used a ten-point scale to measure the patient's sleep quality, defined on a scale from 0 (extremely poor sleep quality) to 10 (extremely good sleep quality). The higher the score was, the better the quality of sleep.

\section{Clinical outcomes}

The primary study outcome measured was the VAS pain score, defined on a scale from 0 (no pain) to 10 (severe pain). In particular, we recorded active and nocturnal VAS pain scores, respectively, which can provide additional useful and detailed information about patients' pain sensations before and after surgery. Night pain was assessed in the morning by nursing staff. In addition, the consumption of analgesic rescue drugs (oxycodone and parecoxib) was recorded, and the doses of oxycodone were expressed in morphine milligram equivalents. We also measured functional parameters, including ROM, Knee Society Score (KSS), and Harris hip score (HHS). In addition, all patients were required to complete a 10point satisfaction questionnaire upon discharge to assess their satisfaction level, defined on a scale from 0 (very dissatisfied) to 10 (extremely satisfied) [26, 27], and the postoperative LOS was recorded. At our institution, patients undergoing arthroplasty are prospectively followed up at 2 weeks and 12 weeks to record their clinical outcomes. For enrolled patients who did not return to their postoperative outpatient appointments, the results were obtained by telephone at the appropriate follow-up points. All complications were also recorded during the first 3 postoperative months.

\section{Statistical analysis}

Distributions of demographic data, baseline data, and primary and secondary outcomes were assessed using measures of central tendency (mean, standard deviation) for quantitative variables and with percentages for qualitative variables. Pearson correlations were performed between the PSQI, ESS, and sleep quality scores with the VAS scores, dose of analgesic rescue drugs, ROM, HHS, KSS, satisfaction score, and LOS. Spearman correlations were also performed between sleep parameters and the number of patients requiring analgesic rescue drugs. An independent sample $t$ test was used to compare patients with and without complications against their PSQI, ESS, and sleep quality measures. All data analyses were performed using SPSS for Windows, Version 19.0 (SPSS Inc., Chicago, IL, USA). Significance was set at $P<0.05$.

\section{Results}

Patient demographics and perioperative characteristics From March 2017 to March 2018, 691 and 724 patients were scheduled to undergo total knee and hip replacement, respectively, at our institution. Among these patients, 191 and 187 were ineligible, while 29 and 14 refuse to participate. Hence, 471 and 523 patients were enrolled in the cohort. No patients were lost or excluded during the follow-up.

The mean patient age (and standard deviation) for those that underwent TKA and THA was $64.28 \pm 9.68$ and $54.85 \pm 13.20$ years, respectively. A total of 114 (24.2\%) and 289 (55.2\%) patients were men among the TKA and THA patients, respectively. The mean patient body mass index (BMI) was $25.81 \pm 3.57$ and $24.10 \pm$ $3.97 \mathrm{~kg} / \mathrm{m}^{2}$, respectively (Table 1 ). Preoperative sleep

Table 1 Baseline, perioperative, and demographic characteristics of the patients

\begin{tabular}{|c|c|c|}
\hline$\overline{\text { Variable }}$ & TKA $(N=471)$ & THA $(N=523)$ \\
\hline \multicolumn{3}{|l|}{ Demographic characteristics } \\
\hline Age (years) & $64.28 \pm 9.68$ & $54.85 \pm 13.20$ \\
\hline Male [no.[\%] of patients] & $114(24.2 \%)$ & $289(55.2 \%)$ \\
\hline Height (m) & $1.57 \pm 0.07$ & $1.61 \pm 0.08$ \\
\hline Weight (kg) & $63.90 \pm 10.19$ & $62.71 \pm 12.01$ \\
\hline BMI $\left(\mathrm{kg} / \mathrm{m}^{2}\right)$ & $25.81 \pm 3.57$ & $24.10 \pm 3.97$ \\
\hline
\end{tabular}

Operated side [no.[\%] of patients]

$\begin{array}{lll}\text { Right } & 221(46.9 \%) & 244(46.6 \%) \\ \text { Left } & 250(53.1 \%) & 279(53.4 \%)\end{array}$

Comorbidities [no.[\%] of patients]

$\begin{array}{lll}\text { Obstructive sleep apnea } & 109(23.1 \%) & 123(23.5 \%) \\ \text { COPD } & 56(11.8 \%) & 65(12.4 \%) \\ \text { Hypertension } & 127(26.9 \%) & 132(25.2 \%) \\ \text { Diabetes mellitus } & 75(15.9 \%) & 87(16.6 \%) \\ \text { Preop. pain and function } & & \\ \text { ROM, } & \\ \text { VAS-night } & 92.15 \pm 13.60 & 89.29 \pm 15.71 \\ \text { VAS-ambulation } & 3.84 \pm 1.41 & 3.20 \pm 1.50 \\ \text { HHS (points) } & 6.37 \pm 1.73 & 5.33 \pm 1.02 \\ \text { KSS (points) } & - & 53.07 \pm 13.33\end{array}$

KSS (points)

$\begin{array}{ll}10.27 \pm 3.77 & 10.11 \pm 3.56 \\ 7.70 \pm 2.31 & 7.36 \pm 2.41 \\ 7.86 \pm 1.39 & 8.19 \pm 3.72\end{array}$

TKA total knee arthroplasty, THA total hip arthroplasty, BMI body mass index, COPD chronic obstruct pulmonary disease, ROM range of motion, VAS visual analog scale, HHS Harris hip score, KSS knee society score, PSQI the Pittsburgh Sleep Quality Index, ESS Epworth Sleepiness Scale 
parameters (i.e., PSQI, ESS, sleep quality scores) were $10.27 \pm 3.77,7.70 \pm 2.31$, and $7.86 \pm 1.39$ for TKA patients, and $10.11 \pm 3.56,7.36 \pm 2.41$, and $8.19 \pm 3.72$ for the THA patients (Table 1).

\section{Pain and clinical outcomes}

Postoperative pain and clinical outcomes are reported in Table 2. Notably, the nocturnal and active pain scores in the TKA group were $3.14 \pm 1.41$ and $4.99 \pm 1.73$ on postoperative day (POD) 1 , and the pain was gradually relieved 3 days, 14 days, and 3 months after surgery. In addition, the pain scores in the THA group showed a similar trend than the TKA group. The average ROM

Table 2 Pain and clinical function outcome postoperation

\begin{tabular}{|c|c|c|}
\hline Variable & TKA $(N=471)$ & THA $(N=523)$ \\
\hline \multicolumn{3}{|l|}{ POD1 } \\
\hline VAS-night & $3.14 \pm 1.41$ & $2.79 \pm 1.71$ \\
\hline VAS-ambulation & $4.99 \pm 1.73$ & $4.11 \pm 1.88$ \\
\hline $\mathrm{ROM}^{\circ}$ & $98.11 \pm 11.29$ & $97.69 \pm 7.47$ \\
\hline HHS (points) & - & $58.38 \pm 13.51$ \\
\hline KSS (points) & $54.03 \pm 13.09$ & - \\
\hline \multicolumn{3}{|l|}{ POD3 } \\
\hline VAS-night & $2.67 \pm 1.29$ & $2.26 \pm 1.35$ \\
\hline VAS-ambulation & $3.93 \pm 1.54$ & $2.67 \pm 1.66$ \\
\hline $\mathrm{ROM}^{\circ}$ & $110.20 \pm 5.96$ & $110.13 \pm 6.07$ \\
\hline HHS (points) & - & $67.00 \pm 11.25$ \\
\hline KSS (points) & $61.49 \pm 11.52$ & - \\
\hline \multicolumn{3}{|l|}{ POD14 } \\
\hline VAS-night & $1.52 \pm 0.93$ & $1.60 \pm 1.06$ \\
\hline VAS-ambulation & $2.18 \pm 1.11$ & $2.23 \pm 1.22$ \\
\hline $\mathrm{ROM}^{\circ}$ & $112.34 \pm 4.63$ & $113.08 \pm 4.83$ \\
\hline HHS (points) & - & $76.65 \pm 8.56$ \\
\hline KSS (points) & $77.32 \pm 7.46$ & - \\
\hline \multicolumn{3}{|l|}{ РОM3 } \\
\hline VAS-night & $0.98 \pm 0.81$ & $1.04 \pm 0.83$ \\
\hline VAS-ambulation & $1.48 \pm 1.13$ & $1.54 \pm 1.10$ \\
\hline $\mathrm{ROM}^{\circ}$ & $110.85 \pm 6.65$ & $112.14 \pm 6.73$ \\
\hline HHS (points) & - & $83.23 \pm 4.64$ \\
\hline KSS (points) & $81.65 \pm 4.51$ & - \\
\hline \multicolumn{3}{|l|}{ Oxycodone } \\
\hline Number of patients requiring & 68 & 31 \\
\hline Dose (morphine eq.) & $7.71 \pm 15.39$ & $3.71 \pm 9.02$ \\
\hline \multicolumn{3}{|l|}{ Parecoxib } \\
\hline Number of patients requiring & 52 & 27 \\
\hline Dose & $5.90 \pm 14.64$ & $3.54 \pm 11.63$ \\
\hline
\end{tabular}

TKA total knee arthroplasty, THA total hip arthroplasty, ROM range of motion, $V A S$ visual analog scale, HHS Harris hip score, KSS knee society score, $P O D$ postoperative day, $P O M$ postoperative month was $98.11 \pm 11.29$ and $97.69 \pm 7.47$ for the TKA and THA groups on POD1 and $110.20 \pm 5.96$ and $110.13 \pm$ 6.07 on POD3, respectively (Table 2 ). The number of patients requiring parecoxib and oxycodone (morphine milligram equivalents) is shown in Table 2. No PE or DVT was observed in any group (Table 3 ). The average satisfaction level of the two groups after joint replacement reached 8.9 points. The average postoperative LOS in the knee replacement group was $4.21 \pm 2.23$ days, while that in the hip replacement group was $4.05 \pm 2.51$ days (Table 3).

\section{Correlation between sleep and clinical parameters}

The correlations between the sleep parameters and postoperative pain, ROM, postoperative LOS, and clinical function outcomes after TKA are presented in Table 4. Before TKA, the PSQI scores were significantly correlated with increased nocturnal $(r=0.123, P=0.008)$ and active $(r=0.118, P=0.010)$ pain scores. In addition, the PSQI scores were significantly correlated with nocturnal $(r=0.098, P=0.034)$ and active $(r=0.127, P=0.006)$ pain scores and ROM $(r=-0.149, P=0.001)$ and KSS $(r=-0.209, P=0.001)$ on POD1. Similarly, sleep quality was significantly correlated with active pain scores $(r=-$ $0.099, P=0.031)$ and ROM $(r=0.129, P=0.005)$ on POD1. There was also a significant correlation between the PSQI and active pain scores measures on POD3 $(r=$ $0.119, P=0.009)$. However, no significant correlations were noted between the sleep parameters and functional outcomes on POD14. In addition, a negative correlation between the active pain scores and ESS scores was noted on POM3 ( $r=-0.091, P=0.049$ ).

The correlations between the sleep parameters and postoperative pain, ROM, postoperative LOS, and clinical function outcomes after THA are presented in Table 4. The PSQI scores were significantly correlated with increased nocturnal $(r=0.111, P=0.011)$ and active

Table 3 Outcomes regarding adverse events, length of hospital stay, and satisfaction level

\begin{tabular}{lll}
\hline Variable & TKA $(N=471)$ & THA $(N=523)$ \\
\hline Symptomatic DVT & 0 & 0 \\
Asymptomatic DVT & 11 & 14 \\
CMVT & 32 & 33 \\
PE & 0 & 0 \\
Superficial infection & 3 & 2 \\
Haematoma & 5 & 6 \\
Wound secretion & 4 & 6 \\
Satisfaction & $8.92 \pm 0.80$ & $8.98 \pm 0.81$ \\
LOS & $4.21 \pm 2.23$ & $4.05 \pm 2.51$ \\
\hline
\end{tabular}

TKA total knee arthroplasty, THA total hip arthroplasty, DVT deep vein thrombosis, CMVT calf muscular vein thrombosis, $P E$ pulmonary embolism, LOS length of stay 
Table 4 Sleep parameters compared with patient reported pain, ROM, and clinical function after surgery

\begin{tabular}{|c|c|c|c|c|c|c|c|c|c|c|c|c|}
\hline \multirow[t]{3}{*}{ Variable } & \multicolumn{6}{|l|}{ TKA } & \multicolumn{6}{|l|}{ THA } \\
\hline & \multicolumn{2}{|l|}{ PSQI } & \multicolumn{2}{|l|}{ ESS } & \multicolumn{2}{|c|}{ Sleep quality } & \multicolumn{2}{|l|}{ PSQI } & \multicolumn{2}{|l|}{ ESS } & \multicolumn{2}{|c|}{ Sleep quality } \\
\hline & rvalue & $p$ value & $r$ value & $p$ value & $r$ value & $p$ value & $r$ value & $p$ value & $r$ value & $p$ value & $r$ value & $p$ value \\
\hline \multicolumn{13}{|l|}{ Preoperation } \\
\hline VAS-night & 0.123 & 0.008 & -0.056 & 0.226 & -0.087 & 0.060 & 0.111 & 0.011 & 0.071 & 0.107 & 0.049 & 0.264 \\
\hline VAS-ambulation & 0.118 & 0.010 & 0.039 & 0.403 & 0.005 & 0.916 & 0.120 & 0.006 & 0.033 & 0.451 & -0.006 & 0.898 \\
\hline $\mathrm{ROM}^{\circ}$ & -0.033 & 0.470 & 0.027 & 0.553 & 0.051 & 0.269 & -0.038 & 0.381 & -0.034 & 0.432 & -0.011 & 0.798 \\
\hline $\mathrm{KSS} / \mathrm{HHS}$ & -0.030 & 0.510 & 0.020 & 0.665 & -0.007 & 0.887 & -0.134 & 0.002 & -0.098 & 0.025 & 0.096 & 0.028 \\
\hline \multicolumn{13}{|l|}{ POD1 } \\
\hline VAS-night & 0.098 & 0.034 & 0.060 & 0.195 & -0.037 & 0.425 & 0.120 & 0.006 & 0.059 & 0.176 & 0.020 & 0.655 \\
\hline VAS-ambulation & 0.127 & 0.006 & 0.018 & 0.692 & -0.099 & 0.031 & 0.088 & 0.043 & 0.060 & 0.169 & -0.006 & 0.893 \\
\hline $\mathrm{ROM}^{\circ}$ & -0.149 & 0.001 & 0.040 & 0.382 & 0.129 & 0.005 & -0.065 & 0.140 & -0.093 & 0.034 & -0.037 & 0.402 \\
\hline $\mathrm{KSS} / \mathrm{HHS}$ & -0.209 & 0.001 & -0.101 & 0.029 & 0.076 & 0.100 & -0.186 & 0.000 & -0.081 & 0.065 & 0.086 & 0.050 \\
\hline \multicolumn{13}{|l|}{ POD3 } \\
\hline VAS-night & 0.111 & 0.016 & 0.072 & 0.118 & -0.077 & 0.094 & 0.133 & 0.002 & 0.019 & 0.662 & -0.095 & 0.030 \\
\hline VAS-ambulation & 0.119 & 0.009 & 0.108 & 0.019 & -0.049 & 0.289 & 0.101 & 0.021 & -0.014 & 0.745 & -0.008 & 0.849 \\
\hline $\mathrm{ROM}^{\circ}$ & 0.031 & 0.498 & 0.027 & 0.558 & 0.029 & 0.527 & -0.002 & 0.969 & 0.000 & 0.994 & -0.058 & 0.185 \\
\hline $\mathrm{KSS} / \mathrm{HHS}$ & -0.054 & 0.239 & -0.088 & 0.057 & -0.034 & 0.461 & -0.123 & 0.005 & -0.029 & 0.507 & 0.071 & 0.103 \\
\hline \multicolumn{13}{|l|}{ POD14 } \\
\hline VAS-night & -0.057 & 0.220 & 0.004 & 0.934 & 0.072 & 0.117 & 0.106 & 0.016 & 0.096 & 0.028 & 0.017 & 0.702 \\
\hline VAS-ambulation & 0.029 & 0.536 & 0.009 & 0.840 & -0.043 & 0.352 & 0.033 & 0.445 & 0.006 & 0.895 & 0.037 & 0.399 \\
\hline $\mathrm{ROM}^{\circ}$ & 0.005 & 0.918 & -0.005 & 0.918 & -0.004 & 0.934 & -0.032 & 0.461 & -0.119 & 0.007 & -0.021 & 0.627 \\
\hline $\mathrm{KSS} / \mathrm{HHS}$ & 0.042 & 0.366 & 0.003 & 0.954 & -0.022 & 0.636 & -0.059 & 0.176 & -0.028 & 0.527 & -0.053 & 0.224 \\
\hline \multicolumn{13}{|l|}{ POM3 } \\
\hline VAS-night & 0.048 & 0.295 & -0.027 & 0.552 & -0.054 & 0.238 & -0.051 & 0.244 & -0.004 & 0.923 & -0.050 & 0.255 \\
\hline VAS-ambulation & -0.072 & 0.120 & -0.091 & 0.049 & 0.044 & 0.345 & -0.048 & 0.270 & -0.030 & 0.491 & -0.046 & 0.295 \\
\hline $\mathrm{ROM}^{\circ}$ & -0.067 & 0.145 & -0.014 & 0.754 & -0.004 & 0.934 & -0.028 & 0.518 & -0.025 & 0.573 & 0.079 & 0.071 \\
\hline $\mathrm{KSS} / \mathrm{HHS}$ & -0.014 & 0.757 & -0.035 & 0.443 & 0.039 & 0.402 & 0.017 & 0.704 & 0.059 & 0.178 & 0.012 & 0.778 \\
\hline Satisfaction & 0.011 & 0.812 & 0.009 & 0.840 & 0.022 & 0.636 & -0.011 & 0.810 & 0.073 & 0.093 & 0.010 & 0.828 \\
\hline LOS & 0.367 & 0.000 & 0.097 & 0.036 & -0.171 & 0.000 & 0.268 & 0.000 & 0.115 & 0.008 & -0.095 & 0.030 \\
\hline
\end{tabular}

TKA total knee arthroplasty, THA total hip arthroplasty, ROM range of motion, VAS visual analog scale, HHS Harris hip score, KSS knee society score, $P O D$ postoperative day, POM postoperative month, PSQI the Pittsburgh Sleep Quality Index, ESS Epworth Sleepiness Scale, LOS length of stay. Significant $p$ values appear in italics

$(r=0.120, P=0.006)$ pain scores and the HHS $(r=-$ $0.134, P=0.002)$ before THA. Similarly, the ESS $(r=-$ $0.134, P=0.002)$ and sleep quantity $(r=-0.098, P=$ $0.025)$ measures were also noted to be significantly correlated with the HHS before THA. In addition, the PSQI scores significantly correlated with the pain scores on POD1 and POD3.

The correlations between sleep parameters and analgesic use are documented in Table 5 . The average doses of parecoxib after knee arthroplasty were significantly correlated with the PSQI scores $(r=0.114, P=0.013)$. In addition, the number of patients requiring parecoxib was also correlated with the PSQI scores in the THA group $(r=0.086, P=0.048)$. However, no significant correlations were noted between the other sleep parameters and consumption of the analgesics.

Moreover, significant correlations were noted between the sleep parameters and postoperative LOS, which indicated that increased postoperative LOS correlated with poor sleep quality (Table 4). However, there were no significant correlations between sleep parameters and perioperative complications, and postoperative satisfaction levels were also not significantly correlated with sleep parameters (Table 6).

\section{Discussion}

The present study is the first to report the correlation between preoperative sleep parameters and postoperative 
Table 5 Sleep parameters compared with the requirement of the rescue treatment after surgery

\begin{tabular}{|c|c|c|c|c|c|c|c|c|c|c|c|c|}
\hline \multirow[t]{3}{*}{ Variable } & \multicolumn{6}{|l|}{ TKA } & \multicolumn{6}{|l|}{ THA } \\
\hline & \multicolumn{2}{|l|}{ PSQI } & \multicolumn{2}{|l|}{ ESS } & \multicolumn{2}{|c|}{ Sleep quality } & \multicolumn{2}{|l|}{ PSQI } & \multicolumn{2}{|l|}{ ESS } & \multicolumn{2}{|c|}{ Sleep quality } \\
\hline & $r$ value & $p$ value & $r$ value & $p$ value & $r$ value & $p$ value & $r$ value & $p$ value & $r$ value & $p$ value & $r$ value & $p$ value \\
\hline \multicolumn{13}{|l|}{ Oxycodone } \\
\hline Number of patients requiring & -0.059 & 0.205 & -0.044 & 0.338 & -0.034 & 0.467 & 0.052 & 0.239 & 0.037 & 0.403 & -0.035 & 0.423 \\
\hline Dose & -0.026 & 0.568 & -0.030 & 0.517 & -0.041 & 0.376 & 0.058 & 0.183 & 0.039 & 0.377 & -0.027 & 0.540 \\
\hline \multicolumn{13}{|l|}{ Parecoxib } \\
\hline Number of patients requiring & 0.090 & 0.052 & -0.007 & 0.887 & -0.039 & 0.396 & 0.086 & 0.048 & 0.077 & 0.078 & -0.073 & 0.095 \\
\hline Dose & 0.114 & 0.013 & -0.014 & 0.768 & -0.057 & 0.216 & 0.074 & 0.091 & 0.079 & 0.071 & -0.030 & 0.489 \\
\hline
\end{tabular}

TKA total knee arthroplasty, THA total hip arthroplasty, PSQI the Pittsburgh Sleep Quality Index, ESS Epworth Sleepiness Scale. Significant $p$ values appear in italics

function. This prospective single-center study revealed that (1) preoperative sleep quality was significantly related to postoperative pain, function, LOS, and analgesic medication consumption following total hip and knee arthroplasty and (2) preoperative sleep parameters did not affect the postoperative complication rate or patient satisfaction scores. Therefore, we believe that preoperative sleep quality can significantly affect postoperative outcomes. Improvements in sleep quality before joint replacement can effectively relieve postoperative pain, improve patient function, and reduce the LOS and analgesic consumption.

Studies have studied the impact of different inventions on postoperative sleep quality [9-11]. Gong et al. examined the effects of sleep quality on early recovery after total knee arthroplasty and noted that patients taking zolpidem achieved significant improvement in sleep and higher levels of satisfaction than those who did not. Patients in the zolpidem group had lower pain scores and took fewer antiemetics than those in the control group. Moreover, a significant correlation between sleep quality and range of motion (ROM) was detected [10]. As such, lowering the incidence of sleep disturbance has the potential to decrease pain and enhance a patient's mental status during daytime hours after TJA, which may improve functional outcomes and hasten postoperative recovery [28]. However, Gong et al. studied the relationship between postoperative sleep quality and postoperative rehabilitation and did not further explore the influence of preoperative sleep quality in patients. Kirksey et al. explored sleep disruption after total knee arthroplasty and the impact of melatonin on sleep and postoperative pain, and exogenous melatonin did not appear to improve postoperative sleep or pain to a significant degree [11].

The dynamic relationship between chronic sleep deficiency and pain thresholds is complicated. However, it is well established that chronic pain conditions are often associated with insufficient sleep, which in turn is a predictor of persistent pain symptoms in both general populations and in individuals with chronic pain [29]. Haack et al. [30] conducted a study and found that activation of the prostaglandin E2 (PGE2) system appeared to be a potential mediator of increased spontaneous pain in response to insufficient sleep. Weingarten et al. conducted a population-based study [31], and the authors concluded that short TSTs were associated with reports of moderate to severe pain. Odegard et al. [32] concluded that sleep restriction reduced the central nervous system (CNS) response to pain, while some of the subjective pain measures indicated hyperalgesia. Recently, Roehrs et al. conducted [15], on the 3- to 4-day preoperative inpatient recovery measures, the sleep extension group reported significantly less average daily pain (4.4 vs. 5.6, $p<0.04$ ) and less daily morphine milligram equivalent intake $(20.3$ vs. $38.6 \mathrm{mg}, p<0.02)$ than the control group [15]. In addition, the PSQI scores were significantly correlated with nocturnal and active pain scores and the consumption of analgesics in the early period after surgery. Our results suggested that a decrease in preoperative sleep quality can affect postoperative pain, increase postoperative pain sensitivity, and increase postoperative analgesic consumption.

The limited ROM after joint replacement is largely due to contributions of postoperative pain, and the postoperative pain threshold is affected by sleep quality. Gong et al. found that the ROM in the zolpidem group was significantly better than that in the control group from POD5 to POD14 [10]. Long et al. compared the ROM after TKA among patients with four forms of insomnia and noted significant differences in active ROM $(p<0.001)$ among patients with the various forms of insomnia (difficulty in staying asleep and non-restorative sleep has increased active ROM in comparison with patients with difficulty in falling asleep and too early awakening) [33]. In the current study, we found that the ROM on POD1 was significantly positively correlated with sleep parameters, but the ROM on POD3 was not related to sleep parameters. A possible cause for the lack of a correlation with ROM on POD3 is that all patients in our medical center were required to try to achieve $110^{\circ} \mathrm{ROM}$ and were discharged home on POD3.

The LOS continues to be a major predictor of cost for TJA [34]. The present study demonstrated that better 


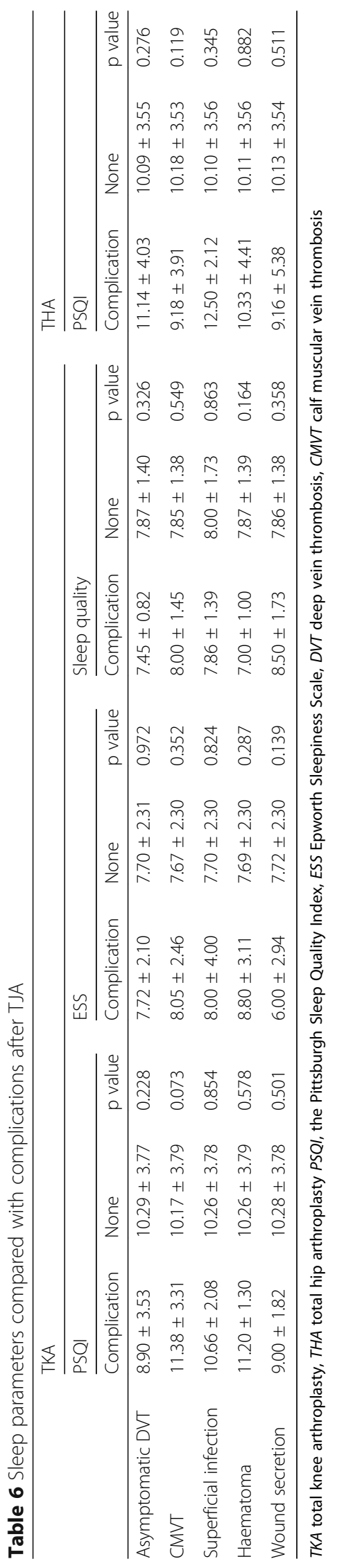




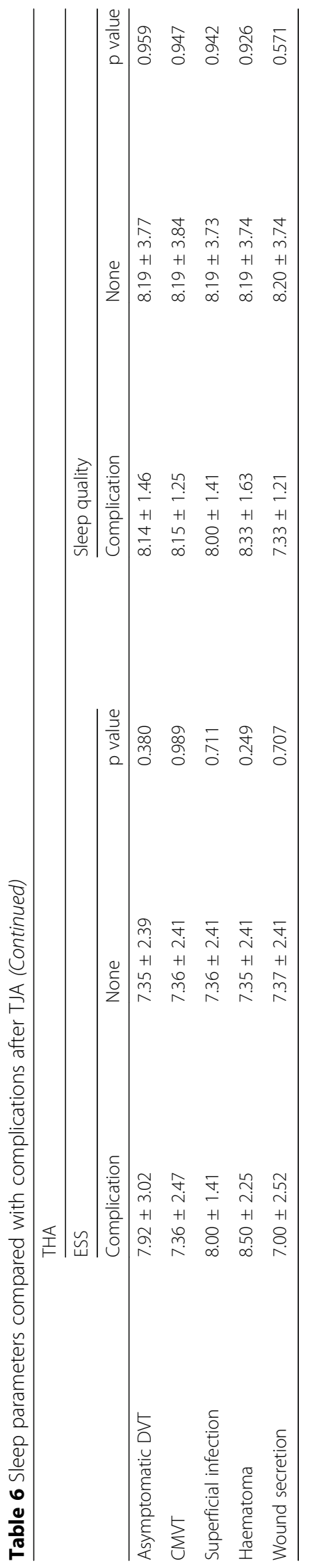


preoperative sleep quality can significantly reduce the LOS for TJA. In addition, Halawi et al. demonstrated that a high preoperative pain level was also a significant multivariable predictor of hospitalization extending beyond 2 days $(p=0.001)$ [35]. Long et al. compared the LOS after TKA among individuals with one of four forms of insomnia (i.e., difficulty in falling asleep, difficulty in staying asleep, non-restorative sleep, too early awakening) and noted that high scores on "Too early awakening" can cause a significantly longer LOS among individuals with various forms of insomnia [33]. The results of our current study indicated that poor preoperative sleep quantity can decrease the postoperative pain threshold, and therefore, the patients required more time to recover from surgical trauma and that these patients had a longer LOS.

There are several limitations to be noted in this study: (1) sleep parameters in the present study were all evaluated via subjective instruments. However, compared to studies using objective measures such as polysomnography (PSG), subjective assessment methods can assess patients' sleep parameters that are closer to their true feelings [33]. (2) Although this is a prospective cohort study, we did not calculate the sample size a priori. We included approximately 500 joint replacements in each group, which is the largest study to research the effects of sleep on total joint replacement.

\section{Conclusion}

Our data revealed correlations between preoperative sleep parameters and postoperative clinical outcomes (i.e., pain, ROM, function, and LOS) in a prospective TJA cohort of 994 patients. Our results indicated that a better sleep quality before the operation could improve the pain threshold, decrease the pain score and analgesic consumption, and reduce the LOS. Considering these proven benefits, we suggest that clinicians should assess the sleep complaints of patients and improve their sleep quality before operation. In addition, these findings point to the importance of and the need for further and larger perspective randomized studies that apply various interventions to improve patients' sleep health during patient preparation in the preoperative period.

\section{Abbreviations}

BMI: Body mass index; CMVT: Calf muscular vein thrombosis; COPD: Chronic obstruct pulmonary disease; DVT: Deep vein thrombosis; ESS: Epworth Sleepiness Scale; HHS: Harris hip score; KSS: Knee Society Score; LOS: Length of stay; PE: Pulmonary embolism; POD: Postoperative day; POM: Postoperative month; PSQI: Pittsburgh Sleep Quality Index; ROM: Range of motion; THA: Total hip arthroplasty; TKA: Total knee arthroplasty; VAS: Visual analog scale

\section{Acknowledgements}

We thank the nursing staff from the Department of Orthopedic Surgery, West China Hospital, and the patients enrolled in this study for their support. We thank American Journal Experts for the editing and writing assistance.

\section{Authors' contributions}

ZYL, LLL, DW, HYW, FXP, and ZKZ designed the study. ZKZ was the principal investigator. ZYL, LLL, and DW were responsible for the trial data collection and outcome analyses. ZYL and LLL were responsible for writing the manuscript. All authors contributed to, read, and approved the final report.

\section{Funding}

This research was funded by the Science \& Technology Department of Sichuan Province Program (2018SZ0135 and 2018SZ0255) and the National Health and Family Planning Commission of the People's Republic of China (program 201302007).

\section{Availability of data and materials}

The datasets analyzed during the current study are available from the corresponding author on reasonable request.

Ethics approval and consent to participate

This retrospective study was approved by the Institutional Review Board of West China Hospital, Sichuan University (ID number 2012-268). All patients provided informed consent for participation.

Consent for publication

Not applicable.

\section{Competing interests}

The authors declare that they have no competing interests.

Received: 11 September 2019 Accepted: 31 October 2019

Published online: 21 November 2019

\section{References}

1. Felson DT. An update on the pathogenesis and epidemiology of osteoarthritis. Radiol Clin N Am. 2004:42(1):1-9 v.

2. Murphy SL, et al. Relationship between fatigue and subsequent physical activity among older adults with symptomatic osteoarthritis. Arthritis Care Res (Hoboken). 2013;65(10):1617-24.

3. Murphy SL, et al. Association between pain, radiographic severity, and centrally-mediated symptoms in women with knee osteoarthritis. Arthritis Care Res. 2011;63(11):1543-9.

4. Parmelee PA, Tighe CA, Dautovich ND. Sleep disturbance in osteoarthritis: linkages with pain, disability, and depressive symptoms. Arthritis Care Res. 2015;67(3):358-65.

5. Redeker NS. Sleep in acute care settings: an integrative review. J Nurs Scholarsh. 2000;32(1):31-8.

6. Luo ZY, et al. Monobloc implants in cementless total hip arthroplasty in patients with Legg-Calve-Perthes disease: a long-term follow-up. BMC Musculoskelet Disord. 2017;18(1):386.

7. Bourne RB, et al. Comparing patient outcomes after THA and TKA: is there a difference? Clin Orthop Relat Res. 2010;468(2):542-6.

8. Chen $\mathrm{AF}$, et al. Prospective evaluation of sleep disturbances after total knee arthroplasty. J Arthroplast. 2016;31(1):330-2.

9. Krenk L, Jennum P, Kehlet H. Postoperative sleep disturbances after zolpidem treatment in fast-track hip and knee replacement. J Clin Sleep Med. 2014;10(3):321-6.

10. Gong L, Wang Z, Fan D. Sleep quality effects recovery after total knee arthroplasty (TKA)--a randomized, double-blind, controlled study. J Arthroplasty. 2015;30(11):1897-901.

11. Kirksey MA, et al. Impact of melatonin on sleep and pain after total knee arthroplasty under regional anesthesia with sedation: a double-blind, randomized, placebo-controlled pilot study. J Arthroplasty. 2015;30(12): 2370-5.

12. Smith MT, et al. The effects of sleep deprivation on pain inhibition and spontaneous pain in women. Sleep. 2007;30(4):494-505.

13. Roehrs T, et al. Sleep loss and REM sleep loss are hyperalgesic. Sleep. 2006; 29(2):145-51.

14. Onen $\mathrm{SH}$, et al. The effects of total sleep deprivation, selective sleep interruption and sleep recovery on pain tolerance thresholds in healthy subjects. J Sleep Res. 2001;10(1):35-42.

15. Roehrs TA, Roth T. Increasing presurgery sleep reduces postsurgery pain and analgesic use following joint replacement: a feasibility study. Sleep Med. 2017;33:109-13. 
16. Parvizi J, Miller AG, Gandhi K. Multimodal pain management after total joint arthroplasty. J Bone Joint Surg Am. 2011;93(11):1075-84.

17. Lei YT, et al. The efficacy and safety of two low-dose peri-operative dexamethasone on pain and recovery following total hip arthroplasty: a randomized controlled trial. Int Orthop. 2018:42(3):499-505.

18. Luo $Z Y$, et al. Oral vs intravenous vs topical tranexamic acid in primary hip arthroplasty: a prospective, randomized, double-blind, controlled study. J Arthroplasty. 2018:33(3):786-93.

19. Yi Z, et al. Tranexamic acid administration in primary total hip arthroplasty: a randomized controlled trial of intravenous combined with topical versus single-dose intravenous administration. J Bone Joint Surg Am. 2016;98(12): 983-91.

20. Wang D, et al. Effect of multiple doses of oral tranexamic acid on haemostasis and inflammatory reaction in total hip arthroplasty: a randomized controlled trial. Thromb Haemost. 2019;119(1):92-103.

21. Healy WL, et al. Complications of total knee arthroplasty: standardized list and definitions of the knee society. Clin Orthop Relat Res. 2013; 471(1):215-20

22. Healy WL, et al. Complications of total hip arthroplasty: standardized list, definitions, and stratification developed by the hip society. Clin Orthop Relat Res. 2016;474(2):357-64.

23. Buysse DJ, et al. The Pittsburgh Sleep Quality Index: a new instrument for psychiatric practice and research. Psychiatry Res. 1989;28(2):193-213.

24. Johns MW. A new method for measuring daytime sleepiness: the Epworth Sleepiness Scale. Sleep. 1991;14(6):540-5.

25. Uchmanowicz I, et al. The relationship between sleep disturbances and quality of life in elderly patients with hypertension. Clin Interv Aging. 2019; 14:155-65.

26. Huang Z, et al. Combination of intravenous and topical application of tranexamic acid in primary total knee arthroplasty: a prospective randomized controlled trial. J Arthroplast. 2014;29(12):2342-6.

27. Coelho PS, Esteves SP. The choice between a five-point and a ten-point scale in the framework of customer satisfaction measurement. Int J Mark Res. 2007:49(3):313-39.

28. Hawker GA, et al. Understanding the pain experience in hip and knee osteoarthritis--an OARSI/OMERACT initiative. Osteoarthr Cartil. 2008;16(4): 415-22.

29. Simpson NS, et al. Chronic exposure to insufficient sleep alters processes of pain habituation and sensitization. Pain. 2018;159(1):33-40.

30. Haack $\mathrm{M}$, et al. Activation of the prostaglandin system in response to sleep loss in healthy humans: potential mediator of increased spontaneous pain. Pain. 2009;145(1-2):136-41.

31. Weingarten JA, et al. Polysomnographic measurement of sleep duration and bodily pain perception in the sleep heart health study. Sleep. 2016; 39(8):1583-9.

32. Odegard SS, et al. The effect of sleep restriction on laser evoked potentials, thermal sensory and pain thresholds and suprathreshold pain in healthy subjects. Clin Neurophysiol. 2015:126(10):1979-87.

33. Long $\mathrm{G}$, et al. Analysis of patients' sleep disorder after total knee arthroplasty-a retrospective study. J Orthop Sci. 2019;24(1):116-20.

34. Sibia US, MacDonald JH, King PJ. Predictors of hospital length of stay in an enhanced recovery after surgery program for primary total hip arthroplasty. J Arthroplast. 2016;31(10):2119-23.

35. Halawi MJ, et al. Preoperative pain level and patient expectation predict hospital length of stay after total hip arthroplasty. J Arthroplast. 2015;30(4): $555-8$.

\section{Publisher's Note}

Springer Nature remains neutral with regard to jurisdictional claims in published maps and institutional affiliations.

Ready to submit your research? Choose BMC and benefit from:

- fast, convenient online submission

- thorough peer review by experienced researchers in your field

- rapid publication on acceptance

- support for research data, including large and complex data types

- gold Open Access which fosters wider collaboration and increased citations

- maximum visibility for your research: over $100 \mathrm{M}$ website views per year

At $\mathrm{BMC}$, research is always in progress.

Learn more biomedcentral.com/submissions 